\title{
INFLUÊNCIA DE FUNGOS MICORRÍZICOS ARBUSCULARES, ASSOCIADA À ADIÇÃO DE COMPOSTOS FENÓLICOS, NO CRESCIMENTO DE MUDAS DE MARACUJ AZEIRO AMARE LO (Passiflora edulis f. flavicarpus) ${ }^{(1)}$
}

\author{
A. C. F. SOARES $S^{(2)} \&$ M. A. MARTINS ${ }^{(3)}$
}

\begin{abstract}
RESUMO
Realizou-se um experi mento em casa de vegetação, com o objetivo de avaliar a influência da inoculação com fungos micorrízicos arbusculares (FMAs), associada à adição de formononetina (nas concentrações de 0,5 e $10 \mu \mathrm{mol} \mathrm{L}-1)$, quercetina e morina (nas concentrações de $0,5,10$ e $15 \mu$ mol $L^{-1}$ ), no crescimento e teor de nutrientes de mudas de maracujazeiro, avaliadas em duas fases: produção das mudas em substrato estéril e após o transplantio para substrato não-estéri l. Utilizaram-se as espécies GI omus clarum (Gc) e Glomus fasciculatum (Gf) e uma população nativa de FMAs (IN) isolada de um plantio de maracujá no município de São J oão da Barra (RJ ). Todos os FMAs avaliados (Gc, Gf e IN) proporcionaram aumentos significativos na produção de matéria seca e no teor de nutrientes na fase de produção de mudas e após o transplantio para substrato não-estéril. A aplicação dos compostos fenólicos teve efeito apenas na fase após o transplantio, destacando-se as plantas não inoculadas que mostraram efeito benéfico da aplicação dos flavonóis quercetina e morina e do isoflavonóide formononetina (apenas na concentração $5 \mu$ mol L-1) na colonização radicular pelos FMAs, indicando que tais compostos estimularam a população nativa de FMAs presente no substrato. Nas plantas inoculadas, não se verificou efeito dos compostos na colonização radicular pelo fungo, mas observou-se efeito positivo em algumas das variáveis analisadas.
\end{abstract}

Termos de indexação: micorrizas, formononetina, quercetina, morina.

(1) Parte da Tese de Doutorado do primeiro autor, apresentada à Universidade Estadual do Norte Fluminense - UENF. Recebido para publicação em abril de 1999 e aprovado em julho de 2000.

(2) Professora Titular do Departamento de Fitotecnia, Escola de Agronomia, Universidade Federal da Bahia - UFBa. CEP $44380-000$ Cruz das Almas (BA).

(3) Professor da UENF. E-mail: marco@uenf.br 


\begin{abstract}
SUMMARY: EFFECTS OF ARBUSCULAR MYCORRHIZAL FUNGI, ASSOCIATED WITH THE ADDITION OF PHENOLIC COMPOUNDS, ON THE GROWTH OF PASSION FRUIT PLANTS (Passiflora edulis f. flavicarpus)
\end{abstract}

\begin{abstract}
An experiment was carried out under green house conditions to evaluate the effects of the inoculation with arbuscular mycorrhizal fungi (AMF), associated with the addition of formononetin $\left(5,10 \mu \mathrm{mol} \mathrm{L}^{-1}\right)$, quercetin and morin $\left(5,10\right.$ and $\left.15 \mu \mathrm{mol} \mathrm{L}^{-1}\right)$, on thegrowth and nutrient content of passion fruit plants in the phase of seedlings cultivated in sterile substrateand after transplanting to a non-steri lesubstrate. Thefungi utilized were Glomus clarum (Gc), Glomus fasciculatum (Gf) and a population of native fungi (IN), which was isolated from a passion fruit plantation in theregion of SãoJ oão da Barra (RJ ). Significant effects on the growth and mineral nutrition of the passion fruit plants were observed for all the AM F utilized (Gc, Gf and IN), during the seedling devel opment and after transplanting to a non-sterile substrata. The phenolic compounds had effects on root colonization and plant growth during the phase of seedl ing grown only in thephase of seedl ings cultivated in a non-sterile substrata, where a beneficial effect of the flavonol s quercetin and morin, and the isoflavonoid formononetin $\left(5 \mu \mathrm{mol} \mathrm{L} \mathrm{L}^{-1}\right)$ on root colonization by AMF, was observed for the non-inoculated seedlings, indicating that these compounds stimulated the native AMF present in the substrata. For the inoculated seedlings with AMF, the flavonoids did not affect the root colonization, but had a positive effect on some variables analyzed.
\end{abstract}

Index terms: mycorrhizae, formononetin, quercetin, morin.

\section{INTRODUÇÃO}

O Brasil é o principal produtor mundial de maracujá amarelo (Passiflora edulis f. flavicarpa Deg.), com uma área plantada de aproximadamente 24.000 ha (Ruggiero et al., 1996). Contudo, o grande entrave para o melhor desenvolvimento da fruticultura é a disponibilidade de mudas selecionadas.

A exploração das associações micorrízicas por meio da inoculação de mudas com fungos micorrízicos arbusculares (FMAs) tem demonstrado grande potencial no desenvolvimento de programas de produção de mudas de boa qualidade para diversas fruteiras (Schubert et al. 1990; Vega-J aizme\& Ázcon, 1991; 1995; Monteiro et al. 1991).

O estabel ecimento deF MAs no sistema radicular das plantas influencia fatores fisiológi cos, tais como: redução da suscetibilidade a certos patógenos (Dehne, 1982; Guillemin et al., 1994), tolerância ao estresse hídrico (Koide, 1985) e alteração da capacidade fotossintética da planta (Brown \& Bethlenfalvay, 1988). Todavia, o principal benefício do fungo para a planta hospedeira, observado quanto ao de melhor desenvol vimento e estado nutricional da planta, está associado à maior absorção de nutrientes, principalmente os de baixa mobilidade no solo, como o fósforo, potássio, zinco e cobre (Marschner \& Dell, 1994). As hifas externas do fungo funcionam como uma extensão do sistema radicular, proporcionando uma área maior para contato com o solo, favorecendo, assim, a maior absorção de nutrientes (Cooper, 1984; Marschner \& Dell, 1994).
A cultura do maracujá necessita da aplicação de doses elevadas de fósforo na fase de produção de mudas e no plantio de pomares, pelo fato de seu sistema radicular apresentar poucos pêlos absorventes e radicelas (Colozzi-Filho \& Carvalho, 1993). O benefício da inoculação com FMA já foi demonstrado por Soares (1994).

Adicional mente, diversos estudos têm demonstrado que compostos fenólicos, especialmente do grupo dos flavonói des, tendem a estimular o desenvolvimento de diversas espécies de FMA (Elias \& Safir, 1987; Tsai \& Phillips, 1991; Bécard et al., 1992; Batista \& Siqueira, 1994) e, conseqüentemente, a colonização do fungo nas raízes (Kaminski et al., 1994; SilvaJ únior \& Siqueira, 1997). Segundo Siqueira et al. (1991), estudos com compostos fenóli cos abrem novas perspectivas para a utilização de FMA, acelerando a col onização e maximizando os benefícios que estes fungos proporcionam às plantas, além de estimularem a população nativa deF MA, destacando o isoflavonói de formononetina como o composto mais ativo.

O presente trabalho objetivou avaliar os efeitos da inoculação com uma população de FMAs nativos isolados de um plantio de maracujá do município de SãoJ oão da Barra, RJ , e das espécies Glomus clarum e Glomus fasciculatum, associadas à adição do isoflavonóide formononetina e dos flavonóis quercetina e morina, em plantas de maracujá amarel o na fase de produção de mudas em substrato estéril e após o transplantio das mudas para substrato não-estéril. 


\section{MATE RIAL E MÉTODOS}

O trabalho foi realizado em casa de vegetação, no período de outubro de 1996 a abril de 1997, na Universidade Estadual do Norte Fluminense. As avaliações foram feitas em duas fases: na fase de produção de mudas em substrato estéril e na fase após o transplantio das mudas para substrato nãoestéril.

\section{Delineamento experimental e descrição dos tratamentos}

Foi utilizado um delineamento experimental em blocos inteiramente casualizados com arranjo fatorial $4 \times 9$ (quatro tratamentos com fungo: Glomus clarum, Glomus fasciculatum, inóculo nativo e testemunha não inoculada; nove tratamentos com compostos: sem composto, formononetina 5 e $10 \mu$ moles $L^{-1}$, quercetina 5,10 e $15 \mu$ moles $L^{-1}$ e morina 5, 10 e 15 umoles $L^{-1}$ ), com três repetições. Este delineamento experimental foi utilizado em ambas as fases de avaliação do trabalho.

\section{Características texturais e químicas do substrato utilizado e adubação}

O substrato utilizado foi uma mistura solo (Cambissolo) peneirado em malha de $4 \mathrm{~mm}$ e areia lavada, na proporção de 1:2 (v/v). Esta mistura (substrato) apresentou as seguintes características texturais e químicas: areia grossa $76 \%$; areia fina $6 \%$; silte 10\%; argila 8\%; carbono $8,0 \mathrm{~g} \mathrm{~kg}^{-1}$; $\mathrm{pH}$ em água (1:2,5) 5,6; $\mathrm{P} 6 \mathrm{mg} \mathrm{dm}^{-3}$ (M ehlich); $\mathrm{P}$ remanescente $52,5 \mathrm{mg} \mathrm{dm}^{-3} ; \mathrm{K}^{0,87} \mathrm{mmol}_{\mathrm{C}} \mathrm{kg}^{-1} ; \mathrm{Ca}$ $16 \mathrm{mmol}_{\mathrm{C}} \mathrm{dm}^{-3}$; $\mathrm{Mg} 11 \mathrm{mmol}_{\mathrm{C}} \mathrm{dm}^{-3}$; $\mathrm{Al} 1 \mathrm{mmol}_{\mathrm{C}} \mathrm{dm}^{-3}$; $\mathrm{H}$ total $18 \mathrm{mmol}_{\mathrm{c}} \mathrm{dm}^{-3}$; $\mathrm{Na} 0,8 \mathrm{mmol}_{\mathrm{C}} \mathrm{dm}^{-1}$. Com base nas características químicas, adicionaram-se individualmente ao substrato de cada saco de polietileno $10 \mathrm{mg} \mathrm{dm}^{-3}$ de $\mathrm{P}$, na forma de $\mathrm{KH}_{2} \mathrm{PO}_{4}$, e $100 \mathrm{mg} \mathrm{dm}^{-3}$ de $\mathrm{K}$, na forma de $\mathrm{KCl}$ em solução aquosa.

\section{FMAs utilizados e preparo dos inóculos}

Foram utilizados os fungos Gl omus cl arum (Gc), Glomus fasciculatum (Gf) euma população defungos nativos (IN) obtida por meio da coleta de uma amostra composta de várias subamostras da camada de $0-15 \mathrm{~cm}$ de solo de um plantio de maracujá, no município de São J oão da Barra, RJ . Os fungos exóticos Glomus clarum e Glomus fasciculatum foram selecionados para este estudo com base nos trabalhos de Soares (1994) e Vega-J aizme \& Àzcon (1991; 1995). Os inóculos de FMAs foram previamente multiplicados em vasos de $3 \mathrm{~kg}$ que continham substrato estéril, utilizando a Brachiaria brizantha como planta hospedeira.

Para a multiplicação da população nativa de FMAs (IN), foi plantada a Braquiaria brizantha (planta hospedeira) no solo coletado no plantio de maracujá. A identificação das espécies de FMAs presentes neste inóculo (IN) foi feita após a multiplicação deste, pelo I nstituto Botânico de São Paulo, Setor de Micologia. Foram identificadas as seguintes espécies na população de fungos nativos (IN): Glomus clarum, Glomus spurcum, Scutellospora fulgida, Glomus macrocarpum, Glomus invermaium, Entrophosfora colombiana, Scutel lospora pel lucida, Acaul ospora appendiculata eScutell ospora heterograma, sendo Gl omus clarum eGlomus spurcum as espécies predominantes.

Como fonte de inóculo, foi utilizada uma mistura de solo que continha esporos e raízes colonizadas pelas espécies de FMAs.

\section{Aplicação dos composto fenólicos}

Inicialmente, os compostos fenólicos foram dissolvidos em um pequeno volume de metanol e, posteriormente, diluídos em água destilada, deforma que a concentração final do metanol, na solução, ficasse em 0,5\%. A concentração dos compostos (determinada com base no peso molecular de cada composto) foi calculada para que, após a aplicação no solo (10 ml da suspensão aplicados na superfície do substrato), fossem obtidas as concentrações finais de 0,5 e $10 \mu \mathrm{M} \mathrm{L}^{-1}$ desubstrato para a formononetina ede $0,5,10$ e $15 \mu \mathrm{M} \mathrm{L}^{-1}$ desubstrato para a quercetina emorina. A formononetina (7-hidróxi-4'-metoxi isoflavona, peso molecular 320,54) foi adquirida pela A pin Chemicals Ltd. (Oxfordshire, Englabd), a quercetina $(3,3 ", 4 ", 5,7-$ pentaidroflavona, peso molecular 338,36$)$ e a morina $(2,3,4,5,7$-pentaidroxiflavona, peso molecular 302,20 ) foram produtos da Sigma, gentilmente fornecidos pelo Departamento de Química da Universidade F ederal de Viçosa (MG).

Nos tratamentos sem composto, adicionaram-se $10 \mathrm{ml}$ de água destilada que continham 0,5\% de metanol. As concentrações testadas foram selecionadas com base nos trabalhos de Bécard et al. (1992) e Silva-J únior \& Siqueira (1997).

\section{Produção das mudas de maracujá}

Para a produção de mudas, as sementes de maracujá amarelo, previamente desinfestadas com uma sol ução de $0,5 \%$ de hi pocl orito de sódio, durante 15 min, e lavadas com água destilada esterilizada, foram pré-germinadas em placas de Petri umedecidas com papel de filtro, em câmara de germinação com temperatura alternada de $20-30^{\circ} \mathrm{C}$, por 10 a 15 dias. Foram utilizados sacos pretos de pol ietileno com $0,5 \mathrm{~kg}$ do substrato esterilizado com brometo de metila (Bromex). A inoculação com os FMAs foi realizada adicionando-se $40 \mathrm{~g}$ do inóculo a aproximadamente $2-3 \mathrm{~cm}$ abaixo do nível do substrato. Foram transplantadas três plântulas de maracujazeiro por saco e, em seguida, foram adicionados os compostos fenólicos.

Uma semana após o plantio, foi feito um desbaste, deixando-se uma planta por saco. As plantas foram 
irrigadas diariamente com água destilada e, a cada 15 dias, foram adicionados $10 \mathrm{ml}$ da seguinte solução nutritiva isenta de fósforo: $2 \mu$ moles $\mathrm{L}^{-1}$ de $\mathrm{Ca}\left(\mathrm{NO}_{3}\right)_{2}$; 2,5 de $\mathrm{KNO}_{3} ; 1$ de $\mathrm{NH}_{4} \mathrm{NO}_{3} ; 1$ de $\mathrm{MgSO}_{4} ; 40$ de FeEDTA; 25 de $_{3} \mathrm{BO}_{3} ; 0,5$ de $\mathrm{KCl}$ e $1 \mathrm{ml}$ de solução de micronutrientes $\left(2 \mu\right.$ moles $\mathrm{L}^{-1}$ de $\mathrm{ZnSO}_{4} \cdot 7 \mathrm{H}_{2} \mathrm{O}$; 0,5 moles $\mathrm{L}^{-1}$ de CuSO $4.5 \mathrm{H}_{2} \mathrm{O} ; 0,5$ de $\mathrm{NaMO}_{4} \cdot 2 \mathrm{H}_{2} \mathrm{O}$; $2 \mu$ moles de $\mathrm{MnSO}_{4} \cdot \mathrm{H}_{2} \mathrm{O}$ ).

As plantas da fase de produção de mudas foram coletadas 80 dias após o plantio (três repetições).

\section{Transplantio das mudas}

$\mathrm{Na}$ época de col eta das mudas de maracujazeiro (primeira fase do trabalho), outras três repetições das mudas foram transplantadas para sacos de pol ietileno que continham $3 \mathrm{~kg}$ do mesmo substrato da primeira fase, porém não esterilizado e com a mesma correção química. Não foi realizada nova adição dos compostos fenóli cos neste substrato, devese considerar, portanto, que houve uma diluição desses composto após o transplantio. As mudas transplantadas para o substrato não esterilizado foram col etadas 70 dias após o transplantio.

\section{Variáveis analisadas após a coleta das plantas, em ambas as fases do trabalho}

Após a col eta das plantas, em ambas as fases de crescimento, foram avaliados o peso da matéria seca da parte aérea e raiz, a percentagem de col onização radicular, através da clarificação das raízes com 10\% $\mathrm{KOH}$, coloração com azul de metila (Koske \& Gemma, 1989) e contagem em placa quadriculada (Giovannetti \& Mosse, 1980), com auxílio de um microscópio estereoscópio com aumento de 40X. Foram determinados os teores de $\mathrm{P}$ e $\mathrm{K}$ da parte aérea. O P foi determinado col orimetricamente pelo método do molibdato e o K por espectrofotometria de emissão de chama, após submeter o material vegetal seco e moído à oxidação pela digestão sulfúrica (Malavolta et al., 1989).
Os resultados foram submetidos à análise de variância e a posterior comparação das médias foi feita pelotestedeTukey, a 5\%, utilizando o programa SAEG-UFV.

\section{RESULTADOS E DISCUSSÃO}

\section{Fase de produção de mudas em substrato estéril}

A aplicação dos compostos fenólicos, nesta fase de produção de mudas em substrato estéril, não teve nenhum efeito significativo $(P<0,05)$ sobre a taxa de colonização micorrízica e sobre o crescimento e teor de nutrientes das mudas de maracujazeiro. O papel dos compostos fenólicos na associação plantaFMA ainda não está bem definido (Werner et al., 1994). Contudo, diversos estudos têm demonstrado que determinados flavonóides, como, por exemplo, a formononetina ea quercetina, tendem a estimular o desenvolvimento de diversas espécies de FMAs (Elias \& Safir, 1987; Tsai \& Phillips, 1991; Becard et al., 1992; Batista \& Siqueira, 1994) e, conseqüentemente, a col onização radicular pelo fungo (Kaminski et al., 1994; Silva-J únior \& Siqueira, 1997).

Neste trabalho, todos os inóculos avaliados demonstraram elevada eficiência em colonizar a planta e promover benefícios no seu crescimento e estado nutricional (Quadro 1), sugerindo que, nestas condições, não há necessidade de se utilizarem os compostos fenólicos para estimular os FMAs e acelerar a col onização radicular pel o fungo.

Todos os inóculos de FMAs utilizados proporcionaram aumentos significativos no crescimento enos teores de nutrientes da parte aérea das mudas de maracujazeiro amarelo, quando comparados aos dos tratamentos sem inoculação (Quadro 1). Observouse um aumento de 317, 397 e $383 \%$ no peso da matéria seca da parte aérea e um aumento de 629,

Quadro 1. Produção de matéria seca da parte aérea (MSPA) e raiz (MSR), teores de fósforo e potássio da parte aérea e colonização das raízes pelos F MAs das mudas de maracujazei ro amarelo, em substrato estéril

\begin{tabular}{lcccc}
\hline \multirow{2}{*}{ Variável } & \multicolumn{3}{c}{ Fungo } \\
\cline { 2 - 5 } & Controle & Inóculo nativo & Glomus clarum & Glomus fasciculatum \\
\hline M SPA (g planta-1) & $0,30^{(1)} \mathrm{C}$ & $1,25 \mathrm{~B}$ & $1,49 \mathrm{~A}$ & $1,45 \mathrm{~A}$ \\
M SR (g planta-1) & $0,07 \mathrm{C}$ & $0,51 \mathrm{~A}$ & $0,52 \mathrm{~A}$ & $0,46 \mathrm{~B}$ \\
P (mg planta-1) & $0,11 \mathrm{D}$ & $1,81 \mathrm{~A}$ & $0,52 \mathrm{C}$ & $1,14 \mathrm{~B}$ \\
K (mg planta-1) & $4,68 \mathrm{C}$ & $21,36 \mathrm{~B}$ & $23,84 \mathrm{~A}$ & $24,27 \mathrm{~A}$ \\
Colonização (\%) & NC(2) & $87,23 \mathrm{~A}$ & $56,93 \mathrm{~B}$ & $60,43 \mathrm{~B}$
\end{tabular}

(1) Os val ores correspondem às médias de todos os tratamentos com compostos. ${ }^{(2)} \mathrm{NC}=$ não col onizada. Médias seguidas pela mesma letra, na mesma linha, dentro da mesma variável, não diferem entre si pelo teste de Tukey a $5 \%$. 
$643,557 \%$ no peso da matéria seca da raiz para a inoculação com IN, Gc e Gf, respectivamente (Quadro 1). Com relação à nutrição das mudas, os teores de $\mathrm{P}$ eK da parte aérea aumentaram em 1.545 e $356 \%$, com ol N ; 373 e $409 \%$, com Gc, e 936 e $419 \%$, com Gf.

A colonização mostrou comportamento distinto entre as espécies, sendo 87, 56 e 60\%, para IN, Gc e Gf, respectivamente. A populaçãonativa, multiplicada previamente com uma planta hospedeira (IN), apresentou maior capacidade de colonização com valores el evados (87\%) e cerca de $30 \%$ superior à das espécies exóticas Gc e Gf. Adicionalmente, as mudas colonizadas pelo I N apresentaram teor de fósforo $1.545 \%$ superior ao das mudas não inoculadas e teores 19 e $59 \%$ superiores aos das mudas colonizadas pelo Gc e Gf, respectivamente, demonstrando el evada eficiência na absorção e, ou, transl ocação de fósforo. Estes dados revelam que o inóculo nativo pode apresentar nítida vantagem sobre os inócul os exóticos, sendo este mais adaptado às condições edafocl imáticas.

Segundo os resultados, o maracujazei ro mostrou elevada dependência micorrízica na fase inicial de desenvolvimento, razão por que as plantas apresentaram efeito significativo da inoculação com FMAs no crescimento vegetativo e no teor de nutrientes. Gerdemann (1975) definiu dependência micorrízica como sendo o grau de dependência da planta pela associação micorrízica para obter o crescimento máximo para determinada condi ção de fertilidade do solo. No presente trabalho, o maracujazeiro apresentou uma dependência micorrízica de $417 \%$, para o IN; $497 \%$, para Gc, e 483\%, para Gf.

As plantas não inoculadas apresentaram sintomas de extrema deficiência de fósforo e um crescimento muito baixo da parte aérea e raiz (Quadro 1). Menge et al. (1978) salientaram que a paralisação do crescimento tem sido observada em mudas de citros plantadas em solo fumigado em diversos viveiros. Estes autores descreveram que a capacidade de absorção de fósforo em solos com baixos níveis de fósforo foi o fator mais importante para o estabel ecimento da dependência micorrízica. Baylis (1970) propôs a teoria de que o comprimento dos pêlos radiculares indicava o grau dedependência micorrízica. Pêlos radiculares curtos indicaram elevada dependência micorrízica, enquanto pêlos radiculares longos indicavam baixa dependência micorrízica, por revelarem maior capacidade de absor ção de fósforo e outros el ementos.

Colozzi-Filho \& Carvalho (1993) também observaram que a cultura do maracujá necessita da aplicação de doses elevadas de fósforo na fase de produção de mudas e no plantio de pomares, pelo fato de seu sistema radicular apresentar poucos pêlos absorventes e radicelas. E ntretanto, o presente trabal ho mostrou que a inoculação com FMAs pode reduzir a necessidade da aplicação de al tas doses de fósforo para a produção de mudas de maracujazeiro de boa qualidade.

\section{Fase pós-transplantio em substrato não-estéril}

Após o transplantio das mudas para substrato não-estéril, observou-se que a aplicação dos compostos fenól icos (na fase de produção de mudas) teve um efeito positivo na col onização, produção de matéria seca da parte aérea, teores de fósforo e potássio das plantas de maracujazeiro (Quadros 2, 3, 4 e5). Observa-se, no quadro 2, que os val ores mais baixos para a colonização ocorreram para o tratamento sem composto e para o tratamento com a formononetina $10 \mu$ moles $\mathrm{L}^{-1}$.

A pós o transplantio das mudas de maracujazeiro para substrato não esterilizado, observou-se que as mudas não inoculadas desenvol vidas em substrato fumigado retomaram o crescimento somente após aproximadamente quatro semanas, apresentando um desenvolvimento ainda significativamente inferior ao das mudas previamente inoculadas com ol N, Gc, e Gf. O intervalo de tempo que ocorreu para a retomada do crescimento das mudas pode estar associado ao tempo necessário para o desenvol vi mento da associação micorrízica, pois estas apresentaram uma percentagem de colonização de $33 \%$, comparada a 72, 65 e 54\%, para I N, Gc, eGf, para o tratamento sem a aplicação dos compostos (Quadro 2).

Os resultados apresentados nos quadros 3, 4, e 5 evidenciam quea inoculação na fase de produção de mudas traz benefícios significativos ao desenvol vimento da parte aér ea eao estado nutricional das mudas após o transplantio.

Adicionalmente, estetrabal ho evidenciou não ser recomendável a prática de fumigação do solo, geralmente utilizada para a eliminação de patógenos. Tal prática elimina toda a microbiota do solo, tornando necessário quese introduzam espécies eficientes de fungos micorrízicos e, ou, se utilizem doses el evadas defósforo para superar a dependência micorrízica do maracujazeiro. Deve-se ressaltar que a fumigação do solo elimina também outros microrganismos importantes para o equilíbrio do ecossistema, como, por exemplo, bactérias diazotróficas e produtoras de substâncias reguladoras de crescimento, cujos efeitos sinergísticos com FMAs têm sido documentados por Graça et al. (1991), em mudas demaracujazeiro; por Paula (1992), em batatadoce, e por Bal ota (1994), em mandioca.

A produção de matéria seca das mudas de maracujazei roapós o transplantioaumentou em 478, 388 e $241 \%$, para as plantas inoculadas com I N, Gce Gf, respectivamente, sem a aplicação dos compostos fenólicos (Quadro 3).

É importantesalientar que o inóculo nativo (I N), que continha uma população de fungos oriunda de um plantio de maracujá da região de São J oão da 
Quadro 2. Colonização radicular pelos FMAs das mudas de maracujazeiro amarelo, após o transplantio para substrato não-estéril

\begin{tabular}{lllll}
\hline \multirow{2}{*}{ Composto(1) } & \multicolumn{3}{c}{ Fungo } \\
\cline { 2 - 5 } & Controle & Inóculo nativo & Glomus clarum & Glomus fasciculatum \\
\cline { 2 - 5 } & & & & \\
S/C & $32,70 \mathrm{Bb}$ & $71,58 \mathrm{Aa}$ & $64,80 \mathrm{Aa}$ & $54,16 \mathrm{Aa}$ \\
F5 & $46,23 \mathrm{Bab}$ & $78,59 \mathrm{Aa}$ & $73,94 \mathrm{ABa}$ & $74,59 \mathrm{ABa}$ \\
F10 & $38,77 \mathrm{Bb}$ & $64,75 \mathrm{Aa}$ & $83,92 \mathrm{Aa}$ & $76,89 \mathrm{Aa}$ \\
Q5 & $79,07 \mathrm{Aa}$ & $75,03 \mathrm{Aa}$ & $67,62 \mathrm{Aa}$ & $74,20 \mathrm{Aa}$ \\
Q10 & $63,08 \mathrm{Aab}$ & $73,93 \mathrm{Aa}$ & $56,37 \mathrm{Aa}$ & $66,58 \mathrm{Aa}$ \\
Q15 & $60,93 \mathrm{ABab}$ & $86,51 \mathrm{Aa}$ & $57,87 \mathrm{Ba}$ & $67,29 \mathrm{ABa}$ \\
M5 & $48,93 \mathrm{Bab}$ & $79,78 \mathrm{Aa}$ & $62,69 \mathrm{ABa}$ & $57,87 \mathrm{ABa}$ \\
M10 & $50,01 \mathrm{Bab}$ & $80,45 \mathrm{Aa}$ & $64,74 \mathrm{ABa}$ & $66,01 \mathrm{ABa}$ \\
M15 & $63,33 \mathrm{Aab}$ & $80,09 \mathrm{Aa}$ & $62,84 \mathrm{Aa}$ & $67,21 \mathrm{Aa}$ \\
\hline
\end{tabular}

(1) Composto: S/C = sem composto; F 5,10 =Formononetina 5 e $10 \mu \mathrm{mol} \mathrm{L}{ }^{-1}$; Q5,10,15 = Quercetina 5, 10 e $15 \mu$ mol L-1; M5,10,15 = Morina 5, 10 e $15 \mu \mathrm{mol} \mathrm{L}^{-1}$. Médias seguidas pela mesma letra maiúscula, na mesma linha, e minúscula, na mesma coluna, não diferem entre si pelo teste de Tukey a $5 \%$.

Quadro 3. Produção de matéria seca da parte aérea das mudas de maracujazeiro amarelo, após o transplantio para substrato não-estéril

\begin{tabular}{|c|c|c|c|c|}
\hline \multirow{2}{*}{ Composto(1) } & \multicolumn{4}{|c|}{ Fungo } \\
\hline & Controle & Inóculo nativo & Glomus clarum & Glomus fasciculatum \\
\hline & & $g$ & $a^{-1}$ & - \\
\hline $\mathrm{S} / \mathrm{C}$ & 2,35 Cab & $13,58 \mathrm{Aa}$ & $11,46 \mathrm{Aa}$ & $8,01 \mathrm{Bab}$ \\
\hline $\begin{array}{l}\text { F5 } \\
\text { F } 10\end{array}$ & $\begin{array}{l}1,55 \mathrm{Cab} \\
0,79 \mathrm{Cb}\end{array}$ & $\begin{array}{r}9,37 \mathrm{Bb} \\
13,35 \mathrm{Aa}\end{array}$ & $\begin{array}{l}12,55 \mathrm{Aa} \\
13,50 \mathrm{Aa}\end{array}$ & $\begin{array}{l}9,62 \mathrm{Abab} \\
9,30 \mathrm{Bab}\end{array}$ \\
\hline $\begin{array}{l}\text { Q5 } \\
\text { Q10 } \\
\text { Q15 }\end{array}$ & $\begin{array}{l}\text { 4,30 Cab } \\
3,42 \mathrm{Cab} \\
3,02 \mathrm{Bab}\end{array}$ & $\begin{array}{l}12,48 \mathrm{Aab} \\
12,71 \mathrm{Aab} \\
11,95 \mathrm{Aab}\end{array}$ & $\begin{array}{l}13,49 \mathrm{Aa} \\
14,30 \mathrm{Aa} \\
12,59 \mathrm{Aa}\end{array}$ & $\begin{array}{l}\text { 8,38 Bab } \\
7,58 \mathrm{Bb} \\
9,93 \mathrm{Aab}\end{array}$ \\
\hline $\begin{array}{l}\text { M5 } \\
\text { M10 } \\
\text { M15 }\end{array}$ & $\begin{array}{l}1,56 \mathrm{Bab} \\
1,50 \mathrm{Bab} \\
4,66 \mathrm{Ca}\end{array}$ & $\begin{array}{l}12,51 \mathrm{Aab} \\
13,20 \mathrm{Aa} \\
12,25 \mathrm{Aab}\end{array}$ & $\begin{array}{l}11,81 \mathrm{Aa} \\
11,34 \mathrm{Aa} \\
11,06 \mathrm{ABa}\end{array}$ & $\begin{array}{c}9,94 \mathrm{Aab} \\
11,29 \mathrm{Aa} \\
8,58 \mathrm{Bab}\end{array}$ \\
\hline
\end{tabular}

(1) Composto: S/C = sem composto; F 5,10 =Formononetina 5 e $10 \mu$ mol L-1; Q5,10,15 = Quercetina 5, 10 e $15 \mu$ mol L-1; M5,10,15 = Morina 5, 10 e $15 \mu \mathrm{mol} \mathrm{L}^{-1}$. Médias seguidas pela mesma letra maiúscula, na mesma linha, e minúscula, na mesma coluna, não diferem entre si pelo teste de Tukey a $5 \%$.

Barra, teve comportamento semel hante ou superior ao comportamento das espécies selecionadas Gc e Gf, demonstrando ser um inóculo eficiente. I sto evidencia que a simples multiplicação da população nativa de FMAs pode ser uma prática viável para o produtor, para melhorar o desenvolvimento da cultura do maracujá. Contudo, a eficiência das populações nativas deve ser testada antes de se adotar tal prática.

Considerando a maior adaptabilidade dos fungos nativos às condições edafocl imáticas, a aval iação da eficiência simbiótica de populações de fungos isolados da própria região de plantio constitui boa estratégia para o desenvol vimento de um programa de produção de mudas micorrizadas, eficientes e sadias.

Saggin-J únior \& Siqueira (1996) citaram diversos estudos que comprovam a elevada eficiência de FMAs ou populações de FMAs nativos isoladas de raízes de cafeei ros, para a produção de mudas desta cultura. Adicionalmente, os FMAs nativos podem exercer grandeinfluência sobre os fungos introduzidos 
Quadro 4. Teor de fósforo da parte aérea de mudas de maracujazeiro amarelo, após o transplantio para substrato não-estéril

\begin{tabular}{|c|c|c|c|c|}
\hline \multirow{2}{*}{ Composto(1) } & \multicolumn{4}{|c|}{ F ungo } \\
\hline & Controle & Inóculo nativo & Glomus clarum & Glomus fasciculatum \\
\hline & & $\mathrm{m}$ & $\operatorname{tat} 2$ & - \\
\hline $\mathrm{S} / \mathrm{C}$ & $4,13 \mathrm{Babc}$ & $15,74 \mathrm{Aa}$ & $13,22 \mathrm{Aa}$ & $11,43 \mathrm{Aa}$ \\
\hline $\begin{array}{l}\text { F5 } \\
\text { F10 }\end{array}$ & $\begin{array}{l}2,29 \mathrm{Cabc} \\
1,33 \mathrm{Bc}\end{array}$ & $\begin{array}{r}9,94 \mathrm{Bb} \\
15,53 \mathrm{Aab}\end{array}$ & $\begin{array}{l}15,01 \mathrm{Aa} \\
14,45 \mathrm{Aa}\end{array}$ & $\begin{array}{l}13,39 \mathrm{ABa} \\
13,77 \mathrm{Aa}\end{array}$ \\
\hline $\begin{array}{l}\text { Q5 } \\
\text { Q10 } \\
\text { Q15 }\end{array}$ & $\begin{array}{l}7,43 \mathrm{Bab} \\
5,23 \mathrm{Babc} \\
4,63 \mathrm{Babc}\end{array}$ & $\begin{array}{l}13,23 \mathrm{Aab} \\
14,58 \mathrm{Aab} \\
13,59 \mathrm{Aab}\end{array}$ & $\begin{array}{l}16,11 \mathrm{Aa} \\
17,37 \mathrm{Aa} \\
14,05 \mathrm{Aa}\end{array}$ & $\begin{array}{l}12,14 \mathrm{ABa} \\
12,72 \mathrm{Aa} \\
14,00 \mathrm{Aa}\end{array}$ \\
\hline $\begin{array}{l}\text { M5 } \\
\text { M10 } \\
\text { M15 }\end{array}$ & $\begin{array}{l}2,34 \mathrm{Babc} \\
2,02 \mathrm{Bbc} \\
7,84 \mathrm{Ba}\end{array}$ & $\begin{array}{l}15,23 \mathrm{Aab} \\
16,69 \mathrm{Aa} \\
13,36 \mathrm{Aab}\end{array}$ & $\begin{array}{l}15,52 \mathrm{Aa} \\
14,31 \mathrm{Aa} \\
13,75 \mathrm{Aa}\end{array}$ & $\begin{array}{l}14,72 \mathrm{Aa} \\
16,69 \mathrm{Aa} \\
13,13 \mathrm{Aa}\end{array}$ \\
\hline
\end{tabular}

(1) Composto: S/C = sem composto; F5,10 =F ormononetina 5 e $10 \mu \mathrm{mol} \mathrm{L}$-1; Q5,10,15 = Quercetina 5, 10 e $15 \mu$ mol L-1; M5,10,15 = Morina 5, 10 e $15 \mu \mathrm{mol} \mathrm{L}{ }^{-1}$. Médias seguidas pela mesma letra maiúscula, na mesma linha, e minúscula, na mesma coluna, não diferem entre si pelo teste de Tukey a 5\%.

Quadro 5. Teor de potássio da parte aérea de mudas de maracujazei ro amarelo, após o transplantio para substrato não-estéril

\begin{tabular}{|c|c|c|c|c|}
\hline \multirow{2}{*}{ Composto(1) } & \multicolumn{4}{|c|}{ Fungo } \\
\hline & Controle & Inóculo nativo & Glomus clarum & Glomus fasciculatum \\
\hline & & $\mathrm{m}$ & $a^{-1}$ & - \\
\hline $\mathrm{S} / \mathrm{C}$ & 90,43 Babc & $237,47 \mathrm{Aa}$ & $262,35 \mathrm{Aa}$ & $219,31 \mathrm{Aa}$ \\
\hline $\begin{array}{l}\text { F5 } \\
\text { F } 10\end{array}$ & $\begin{array}{l}59,15 \mathrm{Cbc} \\
35,36 \mathrm{Bc}\end{array}$ & $\begin{array}{l}162,39 \mathrm{Ba} \\
248,05 \mathrm{Aa}\end{array}$ & $\begin{array}{l}281,85 \mathrm{Aa} \\
255,12 \mathrm{Aa}\end{array}$ & $\begin{array}{l}220,22 \mathrm{Aba} \\
254,39 \mathrm{Aa}\end{array}$ \\
\hline $\begin{array}{l}\text { Q5 } \\
\text { Q10 } \\
\text { Q15 }\end{array}$ & $\begin{array}{l}147,88 \mathrm{Ba} \\
123,47 \mathrm{Cab} \\
104,82 \mathrm{Babc}\end{array}$ & $\begin{array}{l}230,66 \mathrm{Aa} \\
233,07 \mathrm{Ba} \\
233,70 \mathrm{Aa}\end{array}$ & $\begin{array}{l}248,04 \mathrm{Aa} \\
315,97 \mathrm{Aa} \\
224,37 \mathrm{Aa}\end{array}$ & $\begin{array}{l}185,42 \mathrm{Aba} \\
236,53 \mathrm{Ba} \\
247,86 \mathrm{Aa}\end{array}$ \\
\hline $\begin{array}{l}\text { M5 } \\
\text { M10 } \\
\text { M 15 }\end{array}$ & $\begin{array}{r}60,18 \mathrm{Bbc} \\
52,89 \mathrm{Bbc} \\
132,25 \mathrm{Bab}\end{array}$ & $\begin{array}{l}227,76 \mathrm{Aa} \\
222,22 \mathrm{Aa} \\
184,23 \mathrm{ABa}\end{array}$ & $\begin{array}{l}277,71 \mathrm{Aa} \\
274,75 \mathrm{Aa} \\
247,75 \mathrm{Aa}\end{array}$ & $\begin{array}{l}252,40 \mathrm{Aa} \\
271,52 \mathrm{Aa} \\
233,31 \mathrm{Aa}\end{array}$ \\
\hline
\end{tabular}

(1) Composto: S/C = sem composto; F5,10 =Formononetina 5 e $10 \mu$ mol L-1; Q5,10,15 = Quercetina 5, 10 e $15 \mu$ mol L-1; M5,10,15 = Morina 5, 10 e $15 \mu \mathrm{mol} \mathrm{L}^{-1}$. Médias seguidas pela mesma letra maiúscula, na mesma linha, e minúscula, na mesma coluna, não diferem entre si pelo teste de Tukey a $5 \%$.

e a inoculação com espécies eficientes pode ou não trazer benefícios para a planta, em solos com propágulos nativos, dependendo da capacidade de adaptação dessas espécies às condições edafod imáticas e da competitividade com as espécies nativas (Saggin-J únior et al., 1994).

Analisando as condições de transplantio e o período de tempo deste trabalho, o Gc e Gf demonstraram ser eficientes após otransplantio das mudas inoculadas para substratonão-estéril, ou seja, na presença de propágulos nativos.
Nos tratamentos sem inoculação (SI ), a aplicação da quercetina, na dose de $5 \mu$ moles $L^{-1}$, promoveu um aumento significativo na colonização radicular pel os fungos nativos presentes no substrato, quando comparados os tratamentos entre os compostos. A comparação da percentagem de colonização radicular entre os diferentes inóculos (SI, IN , Gc e Gf) mostrou que, nos tratamentos SI , com a aplicação da quercetina $\left(5,10\right.$ e $15 \mu$ moles $\left.L^{-1}\right)$ e morina (15 $\mu$ moles $L^{-1}$ ), a colonizaçãoaumentou para val ores estatisticamentesemel hantes aos da col onização pel os 
inóculos eficientes I N, Gce Gf . A aplicação da morina (5 e $10 \mu$ moles $L^{-1}$ ) e da formononetina ( 5 umoles $\mathrm{L}^{-1}$ ) também aumentou a colonização radicular pelo fungo, atingindo val ores estatisticamente iguais aos obtidos para os inóculos eficientes Gc e Gf, mas inferiores aos obtidos para o IN. Entretanto, a colonização radicular nos tratamentos com a aplicação da quercetina (5, 10 e 15 umoles L-1) e morina (15 $\mu$ moles $L^{-1}$ ) foi mais el evada, semelhante à col onização pelo I N, Gc e Gf.

Tais resultados revelaram que, em substrato com baixoteor defósforo, condição em que o maracujazeiro apresenta elevada dependência micorrízica, tais compostos estimularam a população nativa de fungos, favorecendo a col onização rápida e, com isso, favorecendo o desenvolvimento da planta.

Apesar dea col onização radicular das plantas não inoculadas ter sido semel hante à colonização observada para as plantas inoculadas com I N, Gce Gf, nos tratamentos com a adição da quercetina e morina $\left(5,10\right.$ e $15 \mu$ moles $\left.L^{-1}\right)$ e formononetina (5 umoles $\left.L^{-1}\right)$, a produção de matéria seca e os teores de $\mathrm{P}$ eK das plantas foram significativamente mais baixos, quando comparada entre os inóculos. Isto demonstrou ser a inoculação na fase de produção de mudas com espécies eficientes a mel hor alternativa para obter o bom desenvolvimento e nutrição das mudas após o transplantio para o campo. Contudo, a colonização radicular pelos fungos nas plantascontrole, nas plantas não inoculadas e nas plantas previamente inoculadas ocorreu em épocas diferentes, impedindo comparar a eficiência da colonização pela população de fungos presentes no substrato de transplantio e pelo I N, Gc e Gf.

A comparação entre os compostos, em algumas concentrações do produto, mostrou aumentos significativos no peso da matéria seca da parteaérea e no teores de $P$ e $K$, variando com os fungos (Quadros 3, 4 e5). Nas plantas não inoculadas, apesar detais diferenças não terem si do significativas a 5\%, observou-se uma tendência no aumento de produção de matéria seca e teores de $\mathrm{P}$ e K , nas plantas que receberam quercetina, na concentração de 5 e $10 \mu$ moles $L^{-1}$, e morina, na concentração de $15 \mu$ moles $L^{-1}$, e inferiores nas plantas que receberam a formononetina na concentração de 10 umoles $L^{-1}$, conforme observado para a colonização. Para o I N, observou-se que a formononetina ( $5 \mu$ mol es $L^{-1}$ ) teve um efeito negativo na produção de matéria seca e no teor de fósforo. Adicionalmente, para as plantas inoculadas com Gf, observou-se que os tratamentos com compostos fenóli icos apresentaram um desenvolvimento superior ao do tratamento sem composto.

Silva-J unior \& Siqueira (1997) observaram que a formononetina acel erou a col onização mi corrízica, favoreceu a densidade de arbúscul os evesículas nas raízes desoja e milho, dependendo da época deavaliação, aumentou a produção de matéria seca apenas para a soja, mas não influenciou o acúmulo de nutrientes.
Os resultados revelaram que, em substrato com populações nativas de FMA e com baixo teor de fósforo, como ocorreu após o transplantio das mudas para substrato não-estéril, os compostos fenólicos podem acelerar a colonização pel o fungo, conforme sugerido por Siqueira et al. (1991), e, conseqüentemente, favorecer o desenvol vi mento enutrição das mudas. Por outrolado, na presença de um potencial el evado de inóculo, como ocorreu com os inóculos IN, Gc e Gf, os compostos fenólicos não têm efeitosignificativo na colonização radicular pelos F MAs. A tendência observada no aumento do desenvolvimento enutrição das plantas para o IN e Gf pode ter ocorrido graças ao efeito dos compostos nas hifas externas, arbúsculos e, ou, vesículas, aspectos que variam com a época de avaliação e não foram avaliados neste trabal ho.

\section{CONCLUSÕES}

1. A inoculação com FMAs proporcionou aumentos significativos no crescimento e nos teores de nutrientes da parte aérea das mudas de maracujazeiro, em ambas as fases de crescimento: fase de produção de mudas e fase após o transplantio para substrato não-estéril.

2. O maracujazeiro mostrou elevada dependência mi corrízica na fase inicial de desenvol vimento eapós otransplantio para substratonão-estéril; conseqüentemente, a inoculação com F MAs reduziu significativamentea necessi dade de aplicação de elevadas doses de fósforo em ambas as fases de crescimento da planta.

3. A prática de fumigação do substrato deve ser recomendada para a produção de mudas de maracujazei ro, somente quando se faz a introdução de espécies selecionadas de FMA.

4. Consi derandoa el evada eficiência e capacidade de col onização do I N, semel hante ou superior às das espécies selecionadas Gc e Gf, recomenda-se avaliar a eficiência das populações de fungos nativos, isoladas da própria região de plantio, como estratégia para o desenvolvimento de um programa de produção de mudas de maracujazei ro amarelo.

5. A aplicação de compostos fenólicos não apresentou benefícios na fase de produção de mudas, quando se utilizaram substrato esterilizado e inóculo com um potencial de col onização el evado. Entretanto, em substrato com populações nativas deFMA e com baixo teor de fósforo, como ocorreu após o transplantio das mudas para substrato não-estéril, os compostos fenólicos quercetina e morina $(5,10$ el5 $\mu$ moles $L^{-1}$ ) e formononetina ( $5 \mu$ moles $\left.L^{-1}\right)$, destacando-se a quercetina $\left(5,10 \mu\right.$ moles $\left.^{-1}\right)$ e a morina (15 $\mu$ moles $L^{-1}$ ), podem beneficiar a col onização pelos FMAs nativos presentes no substrato e, conseqüentemente, favorecer o desenvolvimento e nutrição das mudas. 


\section{AGRADECIMENTOS}

À Fundação Estadual do Norte Fluminense (FENORTE ), pel o apoi o financeiro, eà Dra. Sandra Truffen eDra. RosilaineCarrenho, pela identificação das espécies nativas de F MAs.

\section{LITERATURA CITADA}

BALOTA, L.E. Interação de bactérias diazotróficas e fungos micorrízicos arbusculares na cultura da mandioca (Manihot esculenta crantz). I taguaí, Universidade Federal Rural do Rio de J aneiro, 1994. 281p. (Tese de Doutorado)

BAYLIS, G.T.S. Root hairs and phycomycetous mycorrhizas in phosphorus-deficient soil. Plant Soil, 33:713-716, 1970.

BATISTA, M.J . \& SIQUEIRA, J .O. Efeito de flavonóides na germinação de esporos e no crescimento assimbiótico do fungo micorrízico arbuscular Gigaspora gi gantea. R. Bras. Fisiol. Veg., 6:127-134, 1994.

BÉCARD, G.; DOUDS, D.D. \& PFEFFER, P.E. Extensivein vitro growth of vesicular-arbuscular mycorrhizal fungi in the presence of $\mathrm{CO}_{2}$ and flavonoides. Appl. Environ. Microbiol., 58:821-825, 1992.

BROWN, M.S. \& BETHLENFALVAY, G.J . The glycine-glomusrhizobium symbiosis. VII Photosynthetic nutrient-use efficiency in nodulated, mycorrhizal soybeans. Plant Physiol., 86:1292-1297, 1988.

COLOZZI-FILHO, A. \& CARVALHO, S.L.C. Efeitos demicorrízas arbusculares na produção do maracujazeiro a campo. In: CONGRESSO BRASILEIRO DE CIÊNCIA DO SOLO, 24, Goiânia, 1993. Resumos. Goiânia, 1993. p.287-288.

COOPER, K.M. Physiology of VA mycorrhizal association. In: POWELL, C.L. \& BAGYARAJ I, D.J ., eds. VA Mycorrhiza. Boca Raton, CRC Press, 1984. p.155-186.

DEHNE, H.W. Interactions between VA mycorrhizas and plant pathogens. Phytopathology, 72:1115-1119, 1982.

ELIAS, K.S. \& SAFIR, G.R. Hyphal elongation of Glomus fasciculatus in response to root exudates. Appl. Environ. Microbiol., 53:1928-1933, 1987.

GERDEMANN, J.W. Vesicular arbuscular mycorrhiza. In: TORRES, J .G. \& CLARKSON, D.T., eds. The development and function of roots, London, Academic Press, 1975. p.575591.

GUILLEMIN， J.P.; GIANNINAZZI， S.; PEARSON GIANNINAZZI, V. \& MARCHAL, J. Contribution of arbuscular mycorrhizas to biological protection of micropropagated pineapple (Ananas comosus (L.) Merr) against Phytophthora cinnamomi Rands. Agric. Sci. Finland., 3:241-251, 1994.

GIOVANNETTI, M. \& MOSSE, B. An evaluation of techniques for measuring VA mycorrhizal infection in roots. New Phytol., 84:489-500, 1980.
GRAÇA, J .; MACHADO, J .O.; RUGGIERO, C. \& ANDRIOLI, J .L. Eficiência de fungos endomicorrízicos e da bactéria Azospirillum brasiliensesobre o desenvol vimento de mudas do maracujá (Passiflora edul is f. flavicarpa). R. Bras. Frutic., 13:125-130, 1991.

KAMINSKI, J .; FRIES, L.L.M. \& SAFIR, G.R. Efeito da rotação de culturas e da formononetina na col onização de raízes do capim pensacola por fungos MA. In: REUNIÃO BRASILEIRA SOBRE MICORRIZAS. MICORRIZAS E MANEJ O AGROFLORESTAL SUSTENTÁVEL, 5., Florianópolis, 1994. Resumos. Florianópolis, Universidade Federal de Santa Catarina, 1994. p.47.

KOIDE, R.T. The nature of growth depressions in sunflower caused by VA mycorrhizal infection. New Phytol., 99:449462, 1985.

KOSKE, R.E. \& GEMMA, J .N. A modified procedure for staining roots to detect VA mycorrhizas. Mycol. Res., 92:488-505, 1989.

MALAVOLTA, E.; VITTI, G.G. \& OLIVEIRA, S.A. Avaliação do estado nutricional das plantas: princípios e aplicações. Piracicaba, Associação Brasileira para Pesquisa da Potassa e do F osfato, 1989 201p.

MARSCHNER, H. \& DELL, B. Nutrient uptake in mycorrhizal symbiosis. Plant Soil, 159:89-102, 1994.

MENGE, J .A.; J OHNSON, E.L.V. \& PLATT, R.G. Mycorrhizal dependence of several citrus cultivars under threenutrient regimes. New Phytol., 81:553-559, 1978.

MONTEIRO, E.M.S.; MATOS, R.M.B.; PAULA, M.A. \& GUERRA, J .G.M. Micorrizas vesículo-arbusculares em bananeiras: aclimatação e transplante de mudas micropropagadas. In: REUNIÃO BRASILEIRA SOBRE MICORRIZAS, 4., Mendes, 1991. Programa e Resumos. Itaguaí, Empresa Brasileira de Pesquisa Agropecuária, Universidade Federal Rural do Rio de J aneiro, 1991. p.163.

PAULA, M.A. Interação micorrizas vesículo-arbusculares e bactérias diazotróficas em batata-doce (I pomoea batatas L. Lam). Itaguaí, Universidade Federal Rural do Rio de J aneiro, 1992. 168p. (Tese de Doutorado)

RUGGIERO, C.; SÃOJ OSÉ. A.R.; VOLPE, C.A.; OLIVEIRA, J .C.; DURIGAN, J .F.; BAUMGARTNER, J .G.; SILVA, J .R.; NAKAMURA, K.; FERREIRA, M.E.; KAVATI, R. \& PEREIRA, V.P. Maracujá para exportação: aspectos técnicos da produção. Brasília, Ministério da Agricultura, do Abastecimento e da Reforma Agrária, Secretaria de Desenvolvimento Rural, Programa de Apoio à Produção e Exportação de Frutas, Hortaliças, Flores e Plantas Ornamentais, Empresa Brasileira de Pesquisa Agropecuária, 1996. 64p.

SAGGIN-J ÚNIOR, O.J .; SIQUEIRA, J .O.; GUIMARÃES, P.T.G. \& OLIVEIRA, E. Interação fungos micorrízicos versus superfosfato e seus efeitos no crescimento e teores de nutrientes do cafeeiro em solo não fumigado. R. Bras. Ci. Solo, 18:27-36, 1994

SAGGIN-J ÚNIOR, O.J. \& SIQUEIRA, J.O. Micorrizas arbusculares em cafeeiro. In: SIQUEIRA, J .O., ed. Avanços em fundamentos e aplicações de micorrizas. Lavras, Universidade Federal de Lavras, 1996. p.302-303. 
SCHUBERT, A.; MAZZITELLI, M.; ARIUSSO, O. \& EYNARD, I. Effects of vesicular-arbuscular mycorrizal fungi on micropropagated grapevines: I nfluence of endophyte strain, P fertilization, and growth medium. Vitis, 29:5-13, 1990.

SILVA-J ÚNIOR, J.P. \& SIQUEIRA, J.O. Aplicação de formononetina sintética ao solo como estimulante da formação de micorriza no milho e na soja. R. Bras. Fisiol. Veg., 9:35-41, 1997.

SIQUEIRA, J.O.; NAIR, M.G.; HAMMERSCHMIDT, R. \& SAFIR, G.R. Significance of phenolic compounds in plant-soilmicrobial systems. Crit. Rev. Plant Sci., 10:63-121, 1991.

SOARES, I. Associação micorrízica na cultura do maracujá. In: SÃO J OSÉ, A.R. ed. Maracujá-produção e mercado. Vitória da Conquista, Universidade Estadual da Bahia, 1994. p.91-98.
TSAI, S.M. \& PHILLIPS, D.A. Flavonoids released naturally from alfalfa promote development of symbiotic Glomus spores in vitro. Appl. Environ. Microbiol., 57:1485-1488, 1991.

VEGA-J AIZME, M.C. \& ÁZCON, R. Effect of vesicular-arbuscular mycorrizal fungi on pineapple (Ananas comosus L. Merr.) in the Canary Islands. Fruits, 46:47-50, 1991.

VEGA-J AIZME, M.C. \& ÁZCON, R. Responses of some tropical and subtropical cultures to endomycorrhizal fungi. Mycorrhiza, 5:213-217, 1995.

WERNER, D.; BERNARD, S.; GEORGE, E.; ACOBI, E.; KAPE, R.; KOSCH, K.; MULLER, P.; PARNISKE, M.; SCHENCK, P.; SCHIMIDT, P. \& STREIT, W. Competitiveness and comunication for effective inoculation by Rhizobium, Bradyrhizobium and vesicular-arbuscular mycorrhiza fungi. Experientiae, 50:884-889, 1994. 